\title{
L'universalité de la sylloge épigraphique de Gaetano Marini (1742-1815) : le statut philologique des monogrammes du premier millénaire chrétien
}

How universal was Gaetano Marini's (1742-1815) epigraphic sylloge?: the philological status of monograms from the first millenium $C E$

Isabelle Mathian

\section{QpenEdition}

\section{Journals}

Édition électronique

URL : http://journals.openedition.org/cel/1717

DOI : $10.4000 /$ cel. 1717

ISSN : 2262-208X

Éditeur

École du Louvre

Référence électronique

Isabelle Mathian, «L'universalité de la sylloge épigraphique de Gaetano Marini (1742-1815) : le statut philologique des monogrammes du premier millénaire chrétien », Les Cahiers de l'École du Louvre [En ligne], 13 | 2019, mis en ligne le 17 juin 2019, consulté le 17 septembre 2019. URL : http:// journals.openedition.org/cel/1717 ; DOI : 10.4000/cel.1717

Ce document a été généré automatiquement le 17 septembre 2019

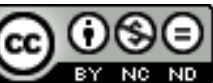

Les Cahiers de l'École du Louvre sont mis à disposition selon les termes de la licence Creative Commons Attribution - Pas d'Utilisation Commerciale - Pas de Modification 4.0 International. 


\section{L'universalité de la sylloge} épigraphique de Gaetano Marini $(1742-1815)$ : le statut philologique
des monogrammes du premier
millénaire chrétien

How universal was Gaetano Marini's (1742-1815) epigraphic sylloge?: the philological status of monograms from the first millenium $C E$

Isabelle Mathian

1 L'imminente publication des Inscriptiones christianae latince et graecae aevi milliarii de Gaetano Marini était annoncée à Rome pour l'année $1797^{1}$. Les cercles érudits attendaient cette sylloge du premier millénaire chrétien car elle cultivait depuis plus de trois décennies l'ambition d'en recueillir, transcrire et annoter toutes les attestations épigraphiques². Elle ne vit jamais le jour. Aujourd'hui enregistrée sous les quatre tomes des mss. Vat. lat. 9071-9074 de la bibliothèque apostolique vaticane ${ }^{3}$, l'œuvre organise la restitution visuelle de 9350 monumenta destinés à ne recevoir d'autre explication que la justesse d'un instrumentum inscriptum ${ }^{4}$ coordonné à la classification de l'objet qui le porte. Cet instrumentum, lorsqu'il prend en compte les caractéristiques des plus divers supports de l'écriture, notamment ceux de l'iconic turn ${ }^{5}$ de l'imagerie tardo-antique et médiévale, devient alors domesticum (fig. 1). 
Fig. 1

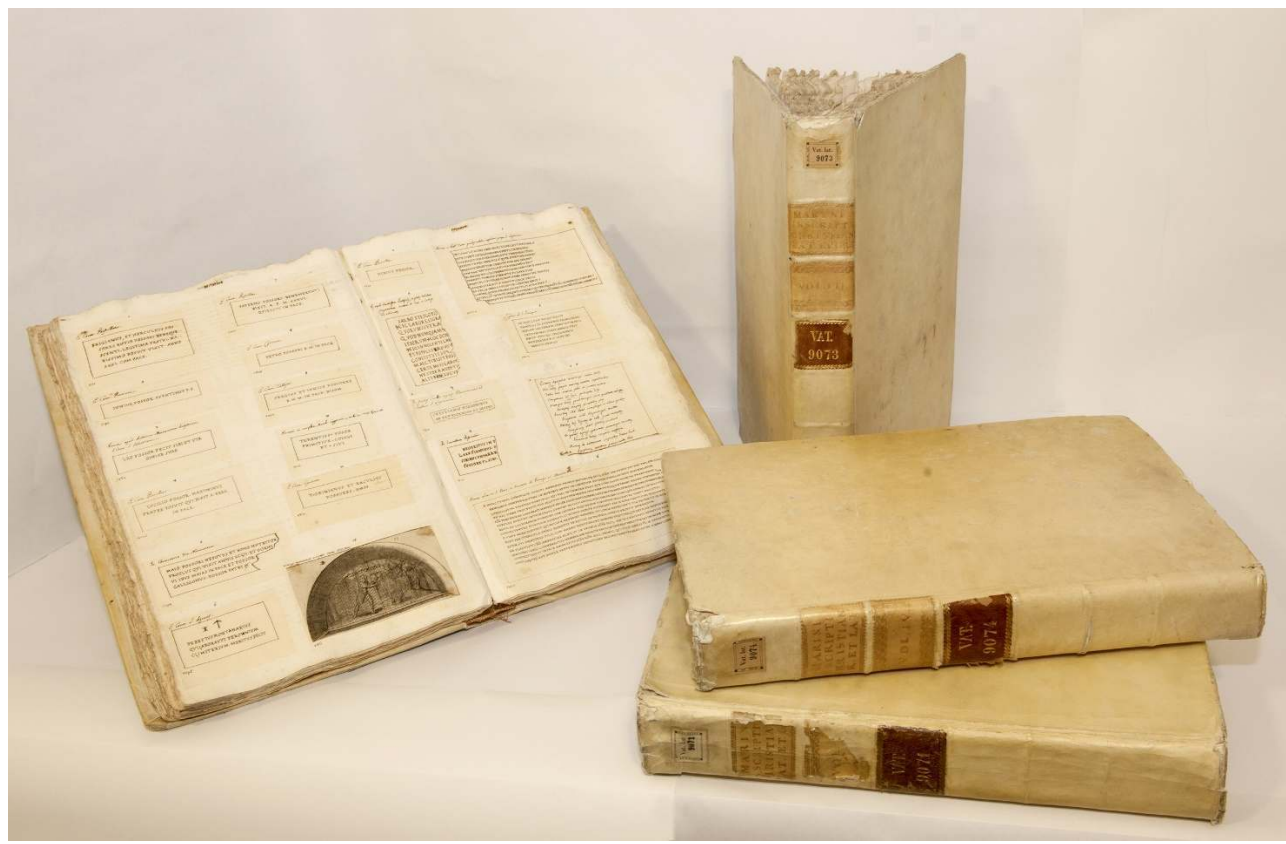

Gaetano Marini, vue d'ensemble des mss. Vat. lat. 9071-9074. Cité du Vatican

(C) Biblioteca Apostolica Vaticana

2 Tout juste diplômé in utroque jure à Ravenne, le jeune Marini était rapidement entré comme Scriptor latinus en 1665 à la bibliothèque vaticane, avant d'en devenir souspréfet (1772), puis préfet (1782) des Archives vaticanes. Il sera aussi président des musées de la bibliothèque (1798), avant d'atteindre la plus haute charge à laquelle un clerc laïc pouvait alors aspirer, celle de premier custode (1800). Les recherches des deux derniers siècles se sont alignées sur l'héroïque fidélité de l'homme à sa triple mission de bibliothécaire, d'archiviste et de conservateur ${ }^{6}$, décédé à Paris le 17 mai 1815 dans un quasi anonymat. Des jours les plus sombres du Traité de Tolentino (1797) jusqu'au dépôt parisien des Archives vaticanes dans la cour de l'Hôtel de Soubise (1808), Marini avait su sacrifier ses recherches épigraphiques à l'impératif de tutelle du patrimoine manuscrit et diplomatique de Rome pour le préserver d'une appropriation incontrôlée de la part des Français. Convaincu que les œuvres ne sauraient jamais être totalement à l'abri de la rapacità e dalla dispersione ${ }^{7}$ qu'impose le cours de l'Histoire, Marini, une fois arrivé à Paris, s'était fait un point d'honneur à taire définitivement la science qu'il avait dispensée dans l'Europe entière pendant un demi-siècle ${ }^{8}$.

L'année même de sa disparition, son premier biographe ${ }^{9}$ notait cependant qu'une société entière de lettrés ne pouvait subvenir à la démesure de l'entreprise des Inscriptiones christianae latince et graecce aevi milliarii. Après qu'Angelo Mai ${ }^{10}(\dagger 1854)$, successeur de Marini à la Vaticane, ait désespérément tenté d'en ordonnancer un premier dépouillement, Giovanni Battista De Rossi († 1894) ne tardait pas à poser son œil averti sur le manuscrit. Celui-ci faisait alors procéder à la reliure des fiches analytiques et bibliographiques que Marini avait accumulées sur chaque occurrence et livrait ainsi les trente-cinq volumes aujourd'hui enregistrés sous les mss. Vat. lat. 9075-9109 ${ }^{11}$. Le projet éditorial des Inscriptiones christiance n'avait donc rien d'improvisé et les fruits de la moisson marinienne avaient fait l'objet d'une colossale sélection. La sentence de De Rossi sur le caractère «farragineux » de l'inédit marinien s'abattait 
malgré tout dans la préface du premier volume des ICUR ${ }^{12}$, cependant qu'Henri Leclercq ${ }^{13}$ restait encore de l'avis, en 1932, que l'œuvre demeurait incompréhensiblement moins digne des sommes scientifiques que l'érudit avait consignées à la postérité.

3 Il ne fait aujourd'hui plus de doutes, notamment grâce aux plus récents travaux de Marco Buonocore ${ }^{14}$, que les Inscriptiones christiance latince et graecee aevi milliarii sont appelées à occuper une place majeure dans la science épigraphique. Et comme le relève à juste titre Antonella Ballardini ${ }^{15}$ dans sa révision des tituli picti présents dans le ms. Vat. lat. 9071, l'historien de l'art tardo-antique et médiéval comprend tout de suite qu'elles s'offrent aujourd'hui comme un musée originel d'une extraordinaire cohérence. L'hypothèse de la concomitance des événements napoléoniens et des coûts d'une édition qui s'annonçait hors-norme, ne résout toutefois pas totalement le problème de la non-publication de l'opera favorita ${ }^{16}$ de l'auteur. C'est ce qu'indiquent en effet quelques lettres récemment publiées dans lesquelles Marini, deux ans avant sa mort, s'inquiète depuis la France que l'on veuille bien voir ses travaux ${ }^{17}$. L'humilité du savant force le respect, mais puisque ni l'intérêt d'Aubin-Louis Millin ( $† 1818)$ pour l'éventuelle acquisition de sa bibliothèque personnelle et du manuscrit, ni celui de la bibliothèque vaticane ne s'étaient concrétisés ${ }^{18}$, il n'était peut-être pas certain que la communauté savante, et un éditeur qui plus est, ait été alors en mesure d'en saisir la valeur.

Ces quelques considérations induiraient donc à situer dans la distance problématique séparant l'inédit d'un chef-d'œuvre, une production qui, il convient de le souligner, n'a encore aucune place dans la synthèse de la bell'antichità et de l'antichità colta auxquelles l'historiographie des années 1770-1800 nous a habitué19. Il est vrai qu'à Rome, les manifestations des primieri christiani - les chrétiens premiers, comme les appelait Cassiano dal Pozzo $(\dagger 1657)$ un siècle plus tôt - étaient encore peu susceptibles de succomber à la mobilisation des apparats du stille surgis du corps de doctrine winckelmannien. Certes, les découvertes des imagines christiance avaient intensifié la valeur des études de philologie textuelle mais, comme tendent maintenant à l'établir plusieurs travaux ${ }^{20}$, les connaissances scientifiques $\mathrm{du}$ XVII $^{\mathrm{e}}$ siècle demeuraient éloignées d'une littéralisation de l'antiquariat dévotionnel en clé apologétique. Si l'historiographie appelle à attendre L'Histoire de l'art par les monumens (1810-1823) de Jean-Baptiste Séroux d'Agincourt $(\dagger 1814)$ pour que l'on découvre aux images chrétiennes des manières hellénisantes dignes d'attention, ce n'est que dans le courant $\mathrm{du}$ XIX $\mathrm{x}^{\mathrm{e}}$ siècle que les milieux archéologiques catholiques romains réagiraient par l'affirmation d'une identité chrétienne conquérante à l'égard de l'Antiquité classique ${ }^{21}$. Dans cet entre-deux, ce n'est pas un hasard, les musées épigraphiques de la Péninsule d'un côté, et singulièrement le musée chrétien et la galerie lapidaire du Vatican, de l'autre, échappaient aux réquisitions du Consulat, puis de l'Empire napoléonien ${ }^{22}$. La contribution des Inscriptiones christiance de Marini se situe précisément à ce croisement décisif des connaissances sur l'Antiquité, à l'heure où les études épigraphiques n'avaient plus à démontrer leur réelle longueur d'avance sur toutes les autres disciplines ${ }^{23}$. Ceci n'est bien sûr pas suffisant pour voir en Marini l'exception historiographique qui renverserait avant l'heure un supposé désert monumental et inscriptionnel parisien. Il convient donc, pour commencer, d'interroger l'état de l' instrumentum que Marini avait laissé à Rome ainsi que la manière dont les Inscriptiones christiance l'avait explicité. 


\section{L'instrumentum inscriptum dans la muséographie pontificale}

Fig. 2

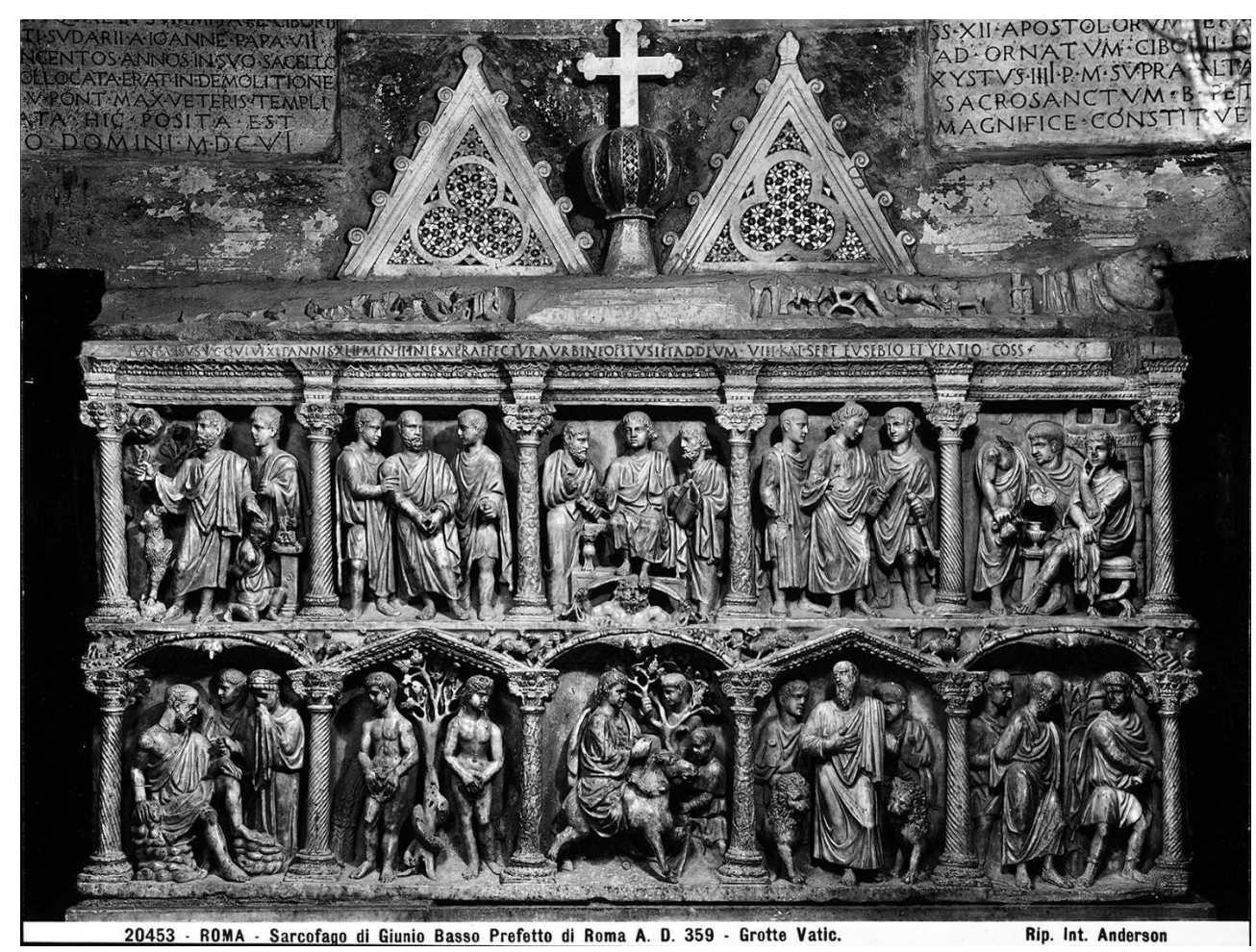

Sarcophage de Junius Bassus dans la crypte de Saint-Pierre en 1890, surmonté.

(C) Alinari - Archivio Anderson

4 En 1606, le pape Paul V (1605-1621) Borghèse signifiait dans une inscription de marbre que la croix du ciborium de l'oratoire proto-médiéval du pape Jean VII († 707) (fig. 2), que l'on démolissait alors pour les travaux de reconstruction de la basilique SaintPierre, seule, en préserverait la mémoire ${ }^{24}$. Fixée au-dessus du sarcophage du Prefectus Urbis Junius Bassus ${ }^{25}$ (†359) mis au jour en 1595 dans le péribole de la confession souterraine de la basilique, la croix de Jean VII pérennisait de sa médiation protomédiévale les derniers siècles de l'Antiquité jusqu'au pontificat en cours. À l'instar de la production épigraphique de l'époque, l'épigraphe de Paul V ignorait l'emprise visuelle du plus insigne des monuments paléochrétiens parce qu'elle renouvelait à l'échelle de l'histoire le legs d'une historicité propre. En 1603, l'édition du Corpus absolutissimum ${ }^{26}$ des inscriptions grecques et latines pensé par Joseph Juste Scaliger (†1609) et achevé par Jan Gruter ( $†$ 1627) l'avait néanmoins précédé, au milieu de quelques 12000 inscriptions, pour restituer l'épitaphe déroulée sur le ruban du sépulcre du préfet Bassus.

On pouvait désormais contempler Rome à l'échelle de la totalité épigraphique de l'empire romain, mais l'inverse restait vrai car l'apostolicité du siège pontifical hérité par Paul V Borghèse présidait à l'unité de l'Église tout entière. D'un côté comme de l'autre, les inscriptions ne pouvaient épuiser la positivité de leurs intentions ostensives puisque l'emploi du titulus, s'il avait été congénital à la théologie politique du nouvel 
horizon constantinien ${ }^{27}$, poursuivait de la force de sa trace la reconnaissance historique, et donc rationnelle, du monde.

L'attachement sémantique de l'inscription à l'imagerie n'était pas encore à l'ordre du jour des discussions scientifiques. L'épigraphie ne cessait cependant de poursuivre et d'affiner la récolte de ses transcriptions : Raffaelle Fabretti ${ }^{28}(\dagger 1700)$ avait accompli un pas décisif à l'intention des moins expérimentés dans les lettres (rudiores) dans une grande Explicatio $^{29}$ sur les inscriptions antiques. Celle-ci couronnait une approche systématique et simplifiée de la titulature impériale latine jusqu'aux minuties du sentiment (affectus) sépulcral et domestique des anciens chrétiens. Ce patricien urbinate, devenu sur le tard surintendant et custode des cimetières sacrés de la papauté, avait aussi revêtu des quelques 432 pièces de sa récolte personnelle tous les murs de la demeure paternelle ${ }^{30}$. Vers la fin du XVII ${ }^{\mathrm{e}}$ siècle, ce premier musée personnel d'épigraphie n'était plus un cabinet de curiosités et sanctionnait au passage un changement politique d'envergure, puisqu'avec la dévolution du duché d'Urbin aux États pontificaux (1631), Rome avait découvert qu'elle pouvait entrer dans une modernité où se passer - enfin ! - de Florence.

Fig. 3

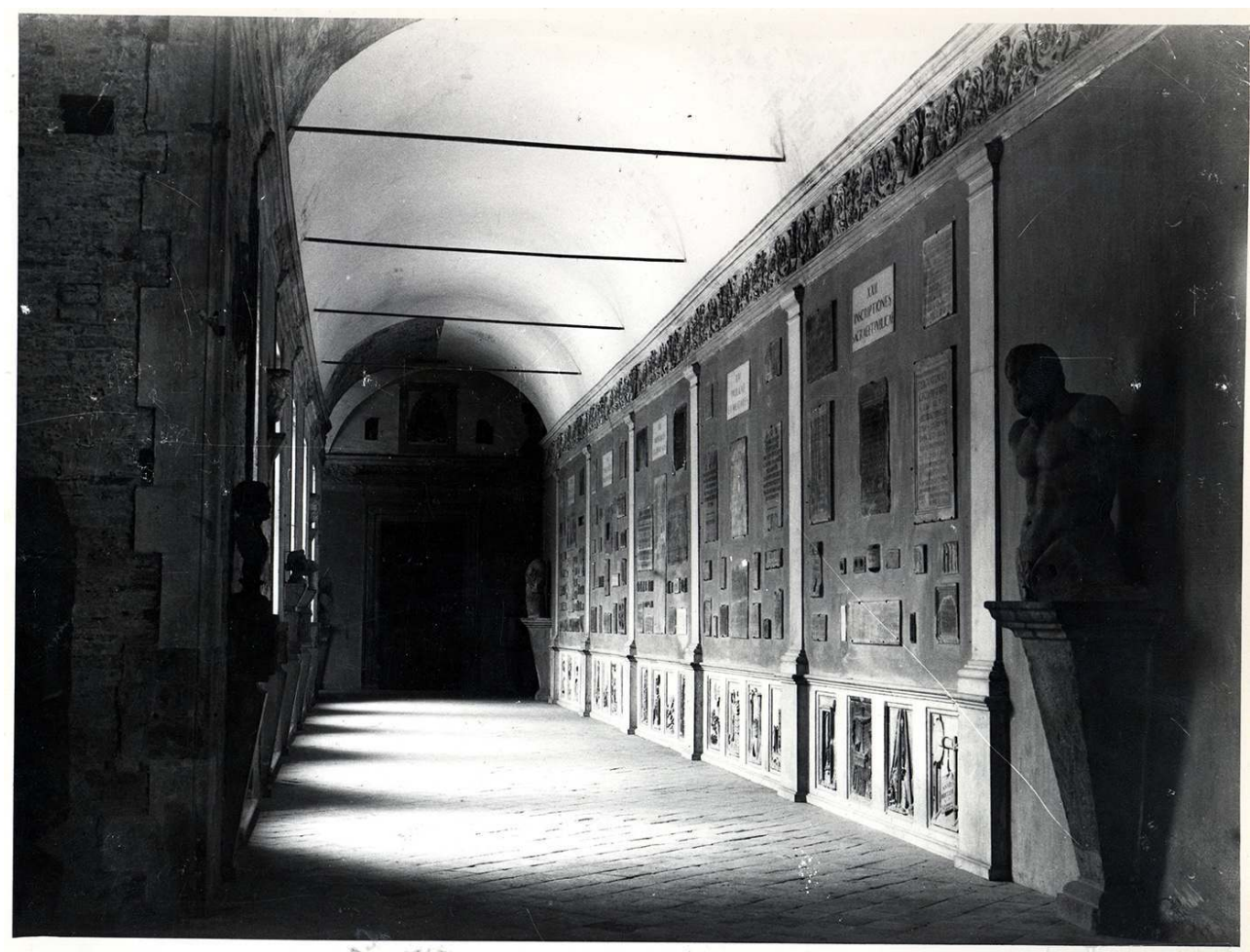

Urbin, Palais ducal, Galleria nazionale delle Marche, gallerie lapidaire (1756-1980).

(c) Galleria Nazionale delle Marche - Urbino

5 Dès 1720, le Museo universale e publico ${ }^{31}$ épigraphique du marquis Scipione Maffei († 1755) parachevait, dans le champ libre d'une image globale enfoncée dans le mur, l'articulation de sens entre dédicaces, dédicants et dédicataires, tandis que les dispositifs historiques de l'écriture s'harmonisaient à une chronologie sociale de la grécité et de la romanité ${ }^{32}$. Les enivrantes théorisations sur le mouseïon ${ }^{33} \mathrm{du}$ savant véronais étaient toutefois loin de trouver un quelconque écho dans le goût des Français. Il restait en effet incompris de quelques «canailles » de l'Académie des inscriptions et 
des belles lettres qui, à Paris, n'avaient pas su saisir sa grandiose proposition d'un musée des inscriptions sous les portiques du Louvre. Qu'importe, en 1756, le cardinal Francesco Stoppani ( $\dagger 1774$ ) pouvait inaugurer un Xystos urbinates (fig. 3) public dans les loges supérieures du palais ducal - devenu pontifical - d'Urbin. La sobre majesté des lieux se prêtait à l'intitulé de cette « palestre rhétorique » qui, pour l'occasion, vantait la synthèse latine constantinienne et universaliste dans la dédicace commémorative du musée au cardinal : FELIX TEMPORUM REPARATIO (Le retour des temps heureux) ${ }^{34}$.

Le musée d'Urbin anticipait de peu l'inauguration, en 1757, du Museo Sacro e Cristiano au premier étage du bras méridional de la bibliothèque vaticane ${ }^{35}$. Au Vatican, il était devenu plus qu'urgent aux yeux du pape Benoît XIV (1740-1758) Lambertini de parer à la plume talentueuse et incrédule de quelques philosophes ${ }^{36}$ : le musée s'ouvrait de la scénographie architecturale d'un frontispice classique et coloré où trônaient, de part et d'autre, les statues assises du pieux rhéteur païen grec du $\mathrm{II}^{\mathrm{e}}$ siècle, Ælius Aristide ${ }^{37}$ ( $†$ 180/185 env.), et du supposé saint docteur de l'Église, aujourd'hui dit le pseudoHippolyte de Rome $^{38}$ (†233/236 env.). Si l'équitable installation des deux antiques mettait un terme à leur pérégrination de l'âge baroque, une étude arithmétique avait démontré que les inscriptions grecques du comput pascal inscrites en l'an 222 sur le dossier du siège s'alignaient sur les calculs astronomiques $\mathrm{du}$ calendrier julien. Vraiment, personne ne se risquerait à l'avenir d'imputer à la chronologie des années de l'Incarnation du Christ d'avoir falsifié l'unicité des temps historiques de l'empire romain, même si on soupçonnait, depuis quelque temps, que Pirro Ligorio ( $† 1583)$ avait commis une fausse inscription grecque sur le socle de la statue d'Aristide et avait resculpté la statue d'Hippolyte.

$\mathrm{Au}$ terme de ces subtiles concessions monumentales et épigraphiques auxquelles la fonction charnière du christianisme avec l'Antiquité gréco-romaine venait de se plier, le pape Clément XIV (1760-1774) Ganganelli envisageait à son tour une trêve théologique de grande ampleur vis-à-vis du paganisme. Marini n'avait pas trente ans et venait de se distinguer aux yeux du pape dans un très structuré Discorso sopra tre candelabri acquistati da S.S. p. Clemente XIV ${ }^{39}$ qui disséquait les leçons du «Signor Winckelmano». Le prétexte était élégant, mais le constat demeurait sans appel à l'égard du Prussien, cette fois définitivement convaincu d'approximations dommageables aux attentes d'une compréhension objective de l'Antiquité : il faudrait à l'avenir éviter des manipulations arbitraires sur des provenances archéologiques que l'on savait douteuses, des divagations iconographiques contraires à la religiosité des Latins corroborée dans l'épigraphie, la publication de planches inexactes et, coup de grâce, des sources textuelles plutôt mal maitrisées. La même année, l'alignement de la collection unique au monde de papyrus ravennates des $\mathrm{v}^{\mathrm{e}} / \mathrm{VII}^{\mathrm{e}}$ siècles sur les parois verticales de la Sala dei Papiri ${ }^{40}$, au premier étage des musées, était confiée à Marini ${ }^{41}$ (fig. $4 \mathrm{a}$ et $4 \mathrm{~b}$ ). Il restait étranger cependant au choix du décor qui devait annoncer la première conjonction entre les arts chrétiens et celle, encore à venir, des arts païens. On ignorait à peu près tout du déchiffrement de ces documents des chancelleries tardoantiques latines écrites en " chinois » - ce qu'il indiquait lui-même en 1805 dans I papiri diplomatici $^{42}$. Pour l'heure, les peintures commandées à Anton Raphael Mengs et Cristoph Unterberger présidaient à ce "paradigme hiéroglyphique ${ }^{43}$ " en version pontificale par le déploiement, sur les contreforts des voûtes, de trompe-l'œil égyptisants d'un côté, et de saint Pierre et Moïse, de l'autre. L'intention n'était pas tant la relative continuité entre l'invention des idéogrammes égyptiens et l'écriture 
hébraïque (biblique), qu'un recentrage sur une allégorie mythique de l'Histoire toute droite issue de la Latinité.

Fig. $4 a$ et $4 b$

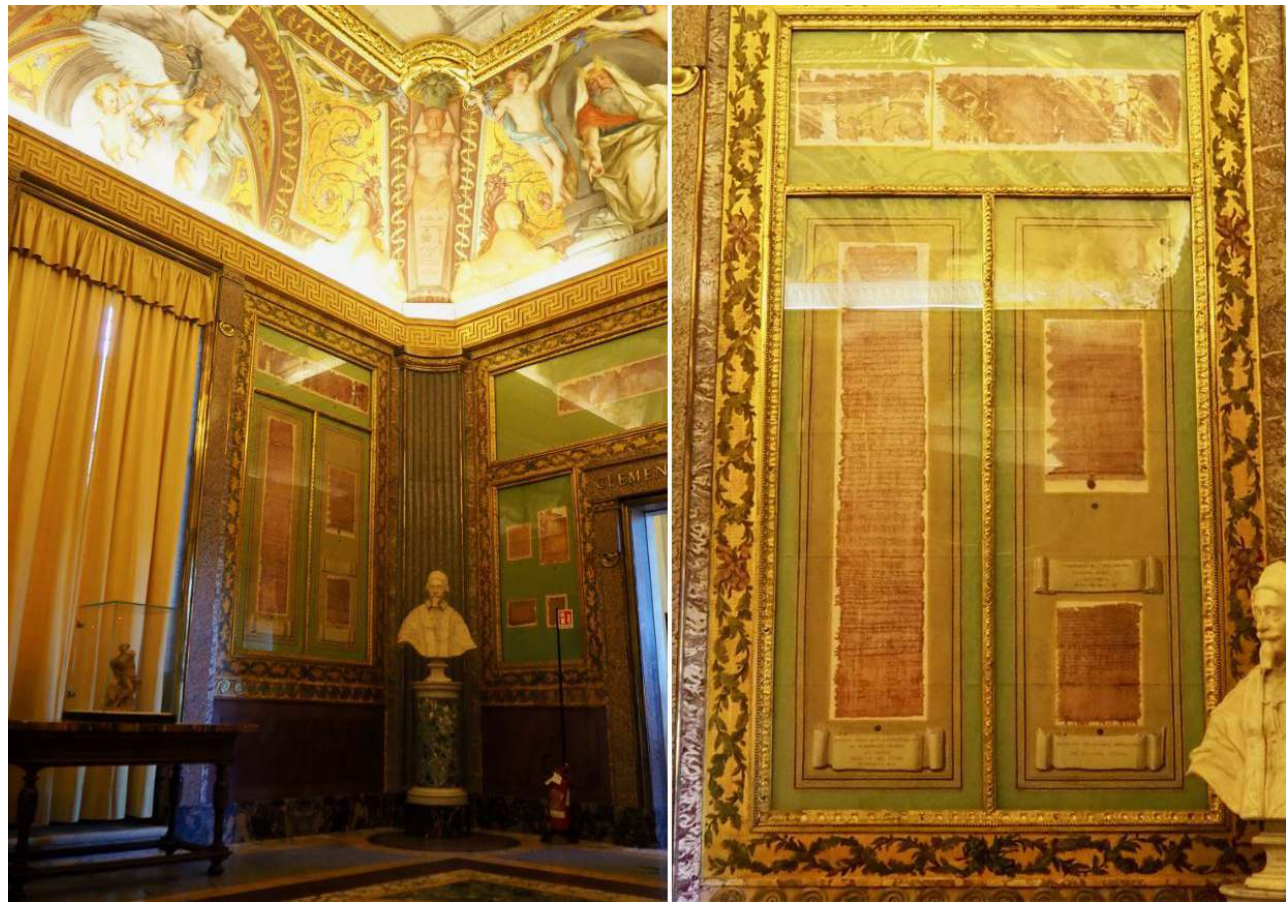

Salle des papyrus (1771-1773).

(c) Isabelle Mathian

6 Les commanditaires ${ }^{44}$ n'ignoraient rien de la réflexion philosophique autour $d u$ constructionnisme mathématisant pré-kantien de Giambattista Vico ( $† 1744)$ - et, non moins, la réception des travaux de William Warburton $(\dagger 1779)^{45}$. La séparation entre les constructions du langage et les contextes linguistiques de la conquête de l'alphabet romain trouvait à ce titre, dans la Salle des papyrus, un aboutissement avec les papyrus ravennates. Mais, au cœur de l'exécution tourmentée de la peinture ${ }^{46}$, l'épitaphe nouvellement découverte ${ }^{47}$ d'un affranchi (libertus), comptable des recettes de la maison impériale julio-claudienne, se glissait au pied d'un grand tableau de Mengs. Depuis l'amitié et l'intense correspondance de Gottfried von Leibniz ( $† 1716)$ avec Fabretti $^{48}$, plus personne ne songeait à exclure l'épigraphie de la médiation entre l'intelligible et le sensible, mais cette fois-ci, le résultat (chrétien) penchait peut-être vers des intentions libérales à peine voilées, voire révolutionnaires.

\section{Le cas des monogrammes chrétiens dans les Inscriptiones christianæ (hors les monogrammes christiques)}

7 À l'instar des hiéroglyphes, les monogrammes avaient eux aussi occupé une place de choix dans les débats philosophiques du fait de l'étrange possession imagée, directe et bidimensionnelle qu'implique celui qui s'en prévalait ${ }^{49}$. La bibliographie n'avait peu ou pas changé depuis qu'un précurseur de l'archéologie chrétienne, Giovanni Ciampini 
$(\dagger 1698)^{50}$ avait fait le tour de ce qui constitue, aujourd'hui encore, le socle philologique de leur définition. Comme en atteste les notes de Marini, il s'en tenait lui aussi aux semeia grecs dont Plutarque fait état dans la vie de Caton le Jeune ${ }^{51}$ - ce que le latin avait traduit de diverses manières : siglae, characterae, litterae monogrammatae. Marini recopiait d'ailleurs une page du De inventione linguarum ${ }^{52}$ dont la paternité était alors attribuée à Raban Maur ( $† 856)$ - mais aujourd'hui à Walafrid Strabon ( $†$ vers 849) pour identifier des congéries de lettres au caractère pictural isolée ${ }^{33}$.

Tout cela n'expliquait pas la décomposition syllabique ou alphabétique des monogrammes chrétiens dont le recours explose, tant sous ses aspects paléographiques qu'épigraphiques, à partir de la seconde moitié $v^{e}$ siècle de notre ère ${ }^{54}$. La recension marinienne, pour l'heure, se contentait de l'option typologique de leur schématisme intrinsèque et, le cas échéant, de leur valeur onomastique.

Fig. 5

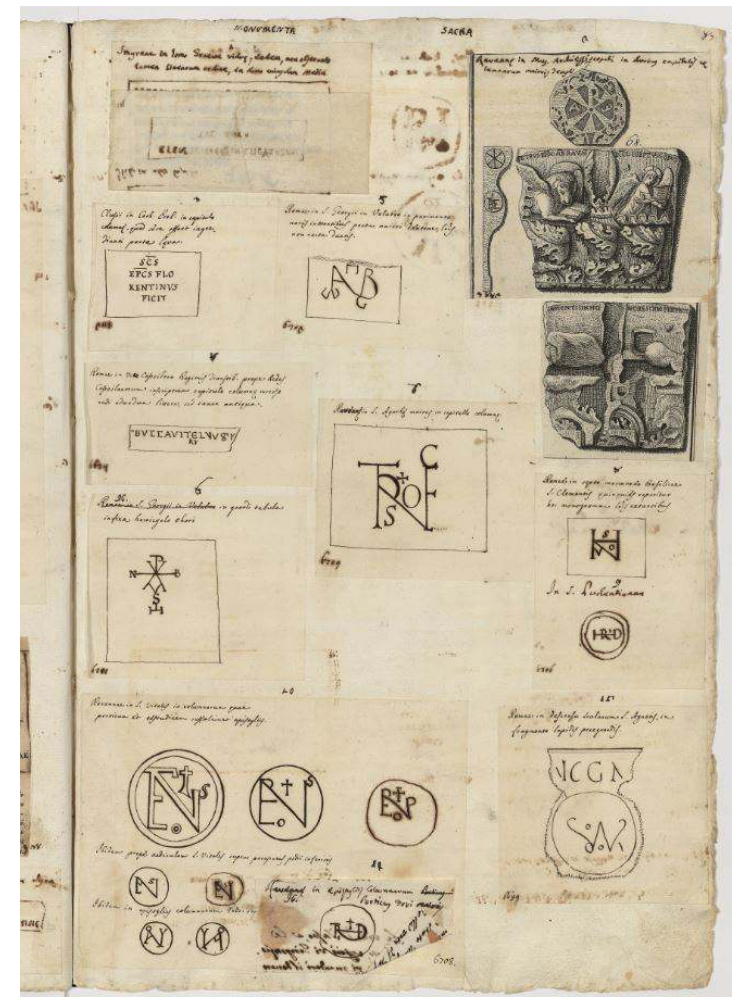

Gaetano Marini (1742-1815), Bibliothèque apostolique vaticane, ms. Vat. lat. 9071, p. 83.

(c) Biblioteca Apostolica Vaticana

Privés de tout commentaire écrit - hormis celui du lieu de conservation - les quatre volumes opèrent à raison d'une numérotation d'une dizaine d'occurrences par page qui, à chaque fois, recommence à la page suivante. Alors que tous les corpora épigraphiques n'utilisaient jusque-là qu'une unique numérotation progressive, l'adoption de ce classement consiste, lui, en une clé-épigraphique-iconographique indépendante de la clé-fiche, rappelée au bas de chaque occurrence : il s'agit, ni plus ni moins, de ce que l'on identifie aujourd'hui comme la méthode relationnelle de nos bases de données modernes. La clé-fiche, en restant totalement indépendante de la cléobjet, autorise le chercheur à une mise-à-jour constante de son corpus. 
Alors qu'un soin extrême est porté à l'instrumentum, l'élément surprenant de la partitio operis reste sans nul doute l'absence de chronologies évidentes ${ }^{55}$, chapitres et souschapitres s'articulent autour d'une double fonction de l'écriture. Celle-ci implique de suivre, en premier lieu, une hiérarchie divine et sacrée du culte chrétien (croix, basiliques, reliques, papes, empereurs et princes etc.) en précisant, au passage, un aspect décisif des datations à travers la mise en parallèle du célèbre calendrier d'Hippolyte de l'an 222 et son adéquation au calendrier pascal de Ravenne pour des années 532-62656. Ce n'est qu'ensuite qu'intervient la seconde grande articulation du classement, à savoir celui de la prosopographie et de l'onomastique sépulcrale (épitaphes) pour démultiplier les axes de compréhension en fonction des formulaires ou des analogies terminologiques. Cette répartition de la matière et du sens doit sa synthèse aux taxinomies héritées de Fabretti et de Maffei, mais elle recouvre, pour emprunter à Gabriel Sanders les mots justes, l'efficience de l'inscription qui « dessine, non pas des fresques panoramiques de l'histoire, mais le cours capricieux de la vie multiple, celle de l'équipage humain des institutions, celle surtout de la masse, de l'homme de la rue en ses moments de suprême importance ${ }^{57}$ ».

À cet égard, les monogrammes obéissent pour la part la plus importante aux Monumenta Sacra du chapitre II de la partie I du ms. Vat. lat. 9071. Ils s'éparpillent en revanche en nombre nettement inférieur dans les divers chapitres des tituli minores gravés, imprimé s ou tracés sur les épitaphes dans les mss. Vat. lat. 9072, 9073, 907458. C'est dans ce groupe que l'on peut identifier d'autres éléments chronologiques, comme par exemple les monogrammes du jeune Alethius (ICUR III, 8748) et du petit Tauro (ICUR X, 26654a), tous deux reconductibles au plein $\mathrm{IV}^{\mathrm{e}}$ siècle, ou encore celui de l'hexamètre épigraphique (CLE 674) d'un inconnu, daté de l'an 386. Le fait est donc que le monogramme fluctue très facilement du décor monumental sacré à la sphère privée de l'infime sépulture - à moins qu'il ne faille, selon notre point de vue, envisager le contraire - dans un acte social de graphicacy ${ }^{59}$, forcément érudit.

10 À titre d'exemple, la page 83 (fig. 5) relative à la section des TEMPLA - AEDES (temples et édifices) du chapitre II, rapporte un ensemble de monogrammes qui succède aux épigraphes des ciboria, elles-mêmes précédées des ambons, et se place avant celle des calices. Leur présence s'inscrit donc dans le classement des supports mobiliers de l'ornement de l'édifice cultuel qui interroge le sens de l'investissement symbolique d'un décor solidaire de l'enveloppe architecturale, mais, à l'évidence, distinct de l'exercice cultuel proprement dit. La deuxième occurrence est tirée d'un découpage de l'édition des Historici Ravennatis (1793) de son ami Camillo Spreti ${ }^{60}(\dagger 1830)$. Il s'agit du chapiteau de l'évêque Pierre II de Ravenne, probablement contemporain du roi Goth, Théodoric († 526). Quant au monogramme de Sainte-Agathe-Majeure - au numéro 6, et toujours à Ravenne - que Bernard de Montfeaucon ( $\uparrow 1741)$ lisait encore comme celui de Titus Cornelius Nepos, il convient pour Marini de retenir la lecture d'Antonio Zirardini (†1785) qui, en 1762, identifiait un autre Petrus Episcopus ${ }^{61}$. Marini est donc un chercheur à jour dans sa bibliographie sur le règne de Théodoric, le Restaurator civitatum, pour lequel il ajoute - en bas de page, au numéro 11 - le monogramme placé sur l'épistyle d'une colonne toujours visible sur la place municipale de Ravenne ${ }^{62}$.

Le cas des monogrammes de Saint-Vital - au numéro 10 - révèle la précision d'une observation in situ qui n'est pas un hasard, puisque Marini a fait ses études à Ravenne. Les trois premiers monogrammes de l'évêque Ecclesius ${ }^{63}$ (†535) situés sur l'abaque des chapiteaux répartis sur le premier niveau de colonnades du chœur y sont distribués en alternance avec ceux d'un certain Ioulianos Argentarios ${ }^{64}$, au niveau même du 
presbyterium. C'est sur la base du papyrus 114 des Papiri diplomatici de Marini ${ }^{65}$ que Friedrich W. Deichmann ${ }^{66}$ relèvera que ce bienfaiteur n'est autre qu'un simple mais richissime vir honestus (honnête homme). La mise en relation entre ce papyrus et les monogrammes de Saint-Vital est cependant suggérée de manière circonspecte par Marini en raison des nombreuses homonymies dans le corps préfectoral ou les milices au service de l'administration impériale ${ }^{67}$. En effet, l'identification de Ioulianos ne va pas sans s'opposer aux orientations juridiques de la novelle justinienne $131^{68}$ de l'année 545 qui veut que seul un vir beatissimus, reverendissimus ou archiepiscopus (un clerc ou un évêque) ait, après l'empereur, le droit de s'afficher dans l'église ${ }^{69}$. La présence de Ioulianos est également digne de note car elle atteste, à l'instar des élites économiques non issues de souche patricienne, d'actes de pieuse et généreuse humilité ${ }^{70}$. La satisfaction de Marini est bien réelle car il sait d'avoir déchiffré, le premier, les caractères paléographiques du nom de ce "grand argentier » qui, souscris en alphabet grec sur l'acte notarié du papyrus ravennate, répondent à la fascinante translittération latinisée et interchangeable de son nom (Y) et de son statut (A).

Rome n'ignore cependant rien des privilèges monogrammatiques du règne de l'empereur Justinien ${ }^{71}$ (527-565) où cette fois-ci - au numéro 8 - Marini se contente de repérer cinq monogrammes sur la clôture de chœur de Saint-Clément ${ }^{72}$. Il convient bien sûr de faire à nouveau mention de la novelle 131 par laquelle l'empereur rétablit aussi le premier rang du Siège apostolique dans un empire qui, lui, siège à Constantinople. Pour le titulaire du monogramme, le pape Jean II (532-535), cette primauté dérive de l'apostolicité du siège du premier apôtre, et non de l'affiliation impériale qu'entend maintenir l'empereur ${ }^{73}$. Dans leur formulation, ces initiales de grande dimension magnifiquement sculptées à Constantinople ${ }^{74}$ entraveraient donc de la volonté pontificale l'entreprise politique de la Renovatio (restauration) impériale.

11 À plus de deux siècles de distance, il conviendrait à ce titre de ne pas exclure l'ambiguïté du D majuscule qui - au numéro 12 - sanctionnait anciennement le monogramme du pape Hadrien Ier (772-795) sur la mosaïque absidale de SaintePudentienne ${ }^{75}$. Il s'agirait autant de l'affirmation antiquisante d'un Dominus théodoricien à la hauteur des interférences doctrinales et politiques d'un autre empereur, Charlemagne ${ }^{76}$, que de la consonne médiane du pape Hadrien. De fait, le monogramme d'un Iohannes, grec, à Saint-Georges au Vélabre - au numéro 6 -, peutêtre sous le pontificat de Jean VIII (872-882), se structure désormais autant sur l'axe cruciforme byzantin que carolingien tout en maintenant -au numéro 7 - une formulation identique plus souple dans les entrelacs cursifs d'un autre Iohannes ${ }^{77}$. 
Fig. 6 et Fig. 7

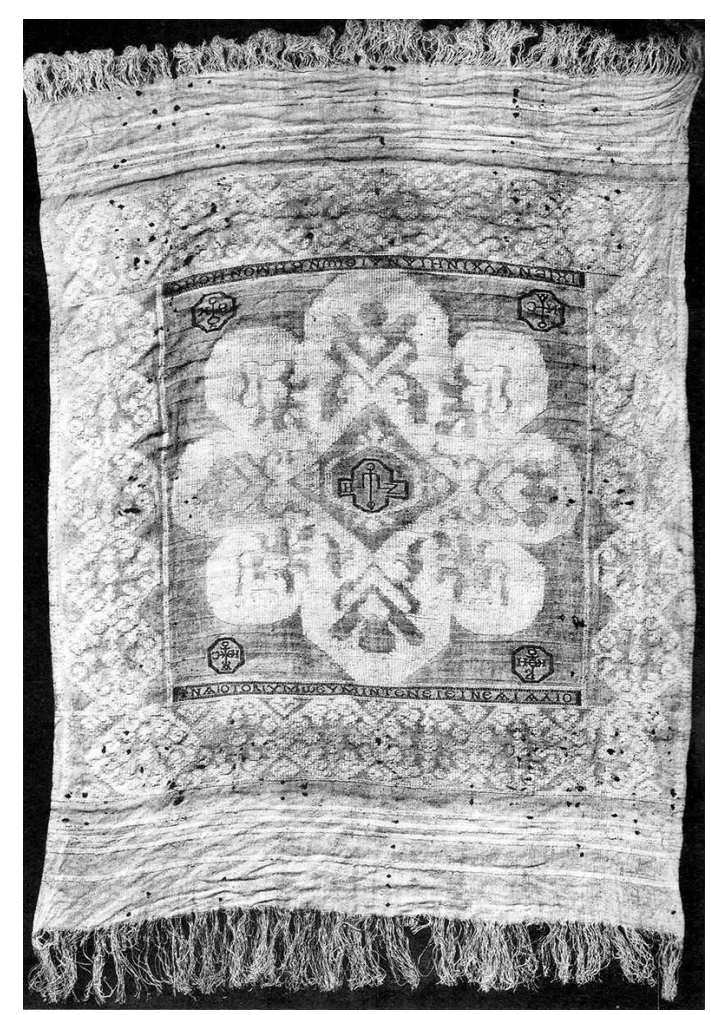

À gauche : Monza, corporal dit «des Apôtres » ou « de Saint Grégoire », vi ${ }^{\mathrm{e}}$-vIl siècle, toile de lin tissée et brodée, Égypte/Constantinople (?), $55 \times 79 \mathrm{~cm}$. Monza. À droite : Jean Mabillon, Iter Italicum litterarium, Paris, 1687, p. 214.

(c)Museo e Tesoro del Duomo di Monza ; ‘ Google Commons

12 Mais attention, Marini insère à la page 91, n. 4 du ms. Vat. lat. 9071, sous la section des DONARIA (dons) liturgiques aux églises, les monogrammes brodés de rouge sur le corporal dit « de saint Grégoire le Grand (590-604) » de la cathédrale de Monza (fig. 6 et 7). Vraisemblablement de peu postérieur au $\mathrm{VI}^{\mathrm{e}}$ siècle, c'est ici l'instrumentum de l'Iter italicum litterarum de Mabillon ${ }^{78}$ qui est retenu pour lire aux encoignures, à n'en pas douter, les monogrammes croisés des quatre évangélistes. Leur dissolution ne sera cependant fixée qu'en 1882 par De Rossi, dans une lettre publiée par Xavier Barbier de Montault dans le Bulletin Monumental ${ }^{79}$. Or, ce que De Rossi ne communique pas, c'est que ce sont en réalité les études diplomatiques de Marini qui ont permis d'élucider l'évolution paléographique qui, à partir $\mathrm{du} \mathrm{VI}^{\mathrm{e}}$ siècle, dispose les lettres grecques latinisées reproduisant la vocale grecque ou par un o surmonté d'un signe diacritique en forme d'accent circonflexe inverséso ${ }^{80}$ La formule placée au centre manifeste en revanche un caractère invocatoire à travers les majuscules grecques $\mathrm{Z} \Omega \mathrm{H} / / \Phi \Omega \Sigma$ (vie lumière), probablement doublé des aspects cryptiques d'une isopsephie du nom latin de Iesous $^{81}$. 
Fig. $8 a$ et $8 b$

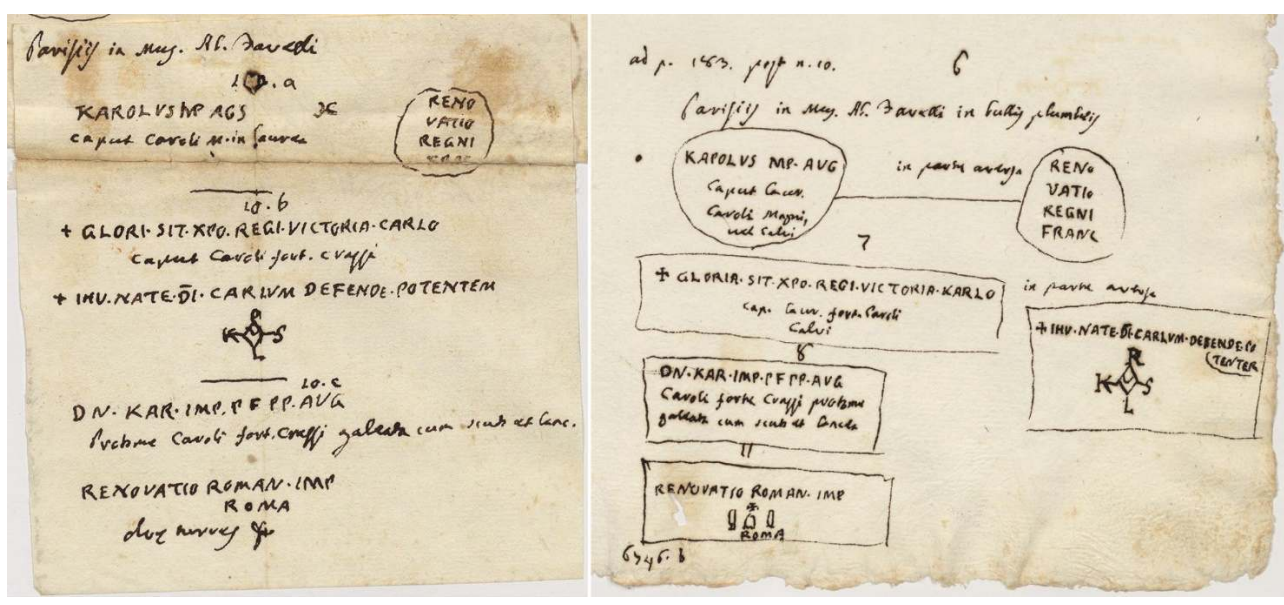

Gaetano Marini (1742-1815), Bibliothèque apostolique vaticane, détail des monogrammes de Charlemagne, ms. Vat. lat. 9071 et ms. Vat. lat. 9074, p. 83, p. 1023.

(C) Biblioteca Apostolica Vaticana

13 Marini se révèle par ailleurs très au fait des localisations, comme à la page $183 \mathrm{du}$ ms. Vat. lat. 9071. Elles lui offrent l'opportunité d'affiner au plus près la généalogie des bulles de plomb des royaumes lombards jusqu'aux réalisations des ateliers de Francie ${ }^{82}$ conservées à Paris dans la collection de l'abbé Fauvel ${ }^{83}$ (fig. 8a et 8 b). L'ensemble de cette titulature romaine impériale est intégrée dans la chronologie aujourd'hui établie des quatre consonnes de KaRoLuS du monogramme cruciforme de Charlemagne rattachées, au centre, dans un losange qui décuple les voyelles A/O/U. Fruit de l'extrême théorisation des enlumineurs de Charlemagne, elle sera suivie, après l'an 800 , de la Renovatio Roman[i - orum] Imperii d'inspiration nettement constantinienne que perpétueront ses proches héritiers ${ }^{84}$. À cette date, toutefois, le monogramme a réellement abandonné sa portée monumentale pour ne gouverner juridiquement que la sphère économique, diplomatique et religieuse. 
Fig. 9

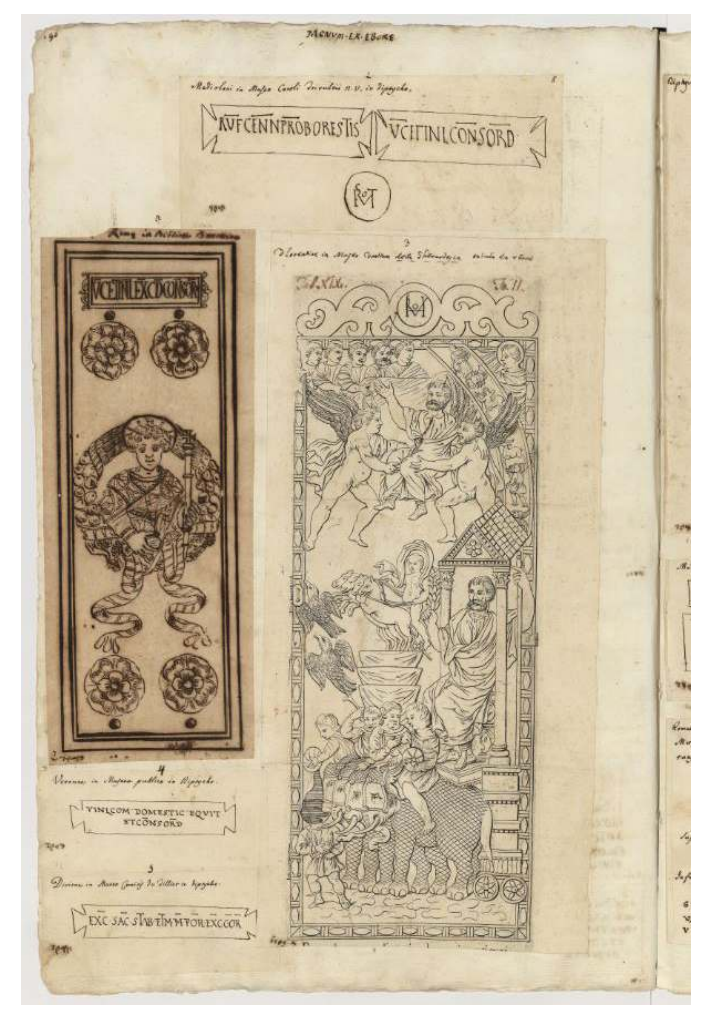

Gaetano Marini (1742-1815), Bibliothèque apostolique vaticane, ms. Vat. lat. 9071, p. 196.

(c) Biblioteca Apostolica Vaticana

14 Ce serait bien évidemment compter sans l'apport des épigraphes des vingt-sept diptyques consulaires recensés des pages 194 à 198 du ms. Vat. lat. 9071. Ils correspondent à un peu moins de la moitié de ceux que recensent Richard Delbrück ${ }^{85}$ en 1929. Le cas de la page 196 (fig. 9) appelle - au numéro 1 - le diptyque d'Oreste ${ }^{86}$, passé en 1781 de la collection Settala de Milan à celle de Carlo Trivulzio. Il appelle également l'inédite transcription du feuillet éburnéen de la bibliothèque Barberini ${ }^{87}$ au numéro 2 - assorti d'un dessin original à l'encre. À partir d'aujourd'hui, l'honneur de cette première restitution doit donc revenir à Marini, c'est-à-dire avant celle, erronée, de la première mention de son plus brillant successeur, Bartolomeo Borghesi $(\dagger 1860)^{88}$.

On ne présente pas l'extraordinaire feuillet du diptyque dit « de la Consécration ${ }^{89}-$ au numéro 3 - aujourd'hui conservé au British Museum, pour lequel Marini se complait à découper la planche XIX d'Anton Francesco Gori ${ }^{90}$ sur laquelle le diptyque figure encore muni de ses attaches métalliques. Après le numéro $4^{91}$, le numéro 5 , relatif au feuillet de la collection parisienne du chevalier du Tilliot, tire sa transcription de celle de Maffei publiée en 1729 dans le cinquième volume de l'Histoire de l'Académie royale des Inscriptions et des Belles Lettres. Marini ne se prononce toutefois pas, contrairement à Maffei, sur le titulaire de ce diptyque qui, dans ce cas, n'est autre que le consul Aréobinde ${ }^{92}$, aujourd'hui logé au musée de Cluny.

Marini a-t-il en tête la question toujours ouverte sur l'usage et l'abstraction politique que représentent ces objets ayant appartenu aux maîtres des milices ou consuls issus de l'aristocratie sénatoriale romaine que le règne finissant de Théodoric anéantit dans le sang $^{93}$ ? Contentons-nous de rappeler, du moins pour les diptyques d'Oreste et « de la 
consécration ", l'institutionnalisation de la trace monogrammatique des chapiteaux ravennates qui, ici, ne peut rivaliser avec la facture encore théodosienne des figures et le rang des propriétaires présumés. Faut-il y voir, comme en émet l'hypothèse Lellia Cracco Ruggini ${ }^{94}$, un passage à des remplois liturgiques chrétiens finalement de très peu postérieurs à leur réalisation?

Marini, cela est certain, savait lire et rien de ce qui avait été écrit ne devait lui rester inconnu. Il est également certain que l'adoption de son double critère typologique lui permet de recouvrer le sens qui primait dans l'esprit des graveurs et des commanditaires chrétiens.

Jamais démentie, sa connaissance parfaite de l'Antiquité n'a rien à voir avec l'idéal normatif de la virilité et de l'héroïsme le plus pur de la Geschichte der Kunst des Altertums. L'érudit procède cependant d'une impulsion tout aussi radicale que celle de Winckelmann dans la redéfinition d'une grammaire visuelle de l'écriture. Il le surpasse peut-être même parce que l'intimité de ce premier classement entraîne derrière lui toute l'histoire. L'acquisition de l'originalité paléochrétienne et proto-médiévale, passée au crible de ce qu'il faut bien définir comme un extraordinaire exercice de philologie visuelle, naît du désir d'universel de son époque tout en restant, à l'instar de son amico carissimo Séroux d'Agincourt, la fille légitime de l'empire romain.

Il est certain que nous avons-là un musée qui ne résistera pas à la redistribution complète qui va avoir lieu au siècle suivant. L'étude reste à mener. Dans deux lettres datées des 9 septembre et 21 novembre $1811^{95}$, le commissaire de guerre de l'armée d'Italie en poste à Vérone, Étienne Auguste Siauve ( $† 1812)$, adressait à Marini la proposition de le voir désigné pour la formation d'un "Lapidaire de la capitale de l'Empire ", enfin digne des progrès que son pays accomplissait. La réponse de Marini, au vu du « déplaisir que [son] séjour à Paris lui cause », ne se fit pas attendre.

\section{NOTES}

1. Antonio Coppi, Notizie sulla vita e sulle opere di Monsignor Gaetano Marini, Rome, 1815, p. 108-109.

2. En page de garde du ms. Vat. lat. 9071 , le titre complet est : Inscriptiones christianae latinæ et graecæ ævi milliarii conlegit, digessit, adnotationibus audit Caietanus Marinus a Bibliotheca Vaticana item Scrinis Sedis Apostolicae.

3. Les quatre manuscrits sont consultables en basse définition sur le site: http://digi.vatlib.it [03/12/2018].

4. Le terme moderne latin d'instrumentum, dans le sens d'un appareil écrit qui dresse conjointement le constat intrinsèque de l'objet et de son sens, apparaît au XIII ${ }^{\mathrm{e}}$ siècle. Pour les études épigraphiques, on peut se reporter à Alison Cooley, The Cambridge Manual of Epigraphy, Cambridge, Cambridge University Press, 2012, pp. 82-84. Voir surtout Silvio Panciera, «Un gruppo romano per lo studio dell'instrumentum domesticum», dans S. Panciera, Epigrafi, epigrafia, epigrafisti : scritti vari editi e inediti, (1956-2005), Rome, Edizioni Quasar, 2006, t. II, pp. 1789-1794; ainsi que Marc Mayer i Olivé, Consideraciones sobre las diversas funciones de la 
inscrptiones didascàlicas o explicativas en el Instrumentum inscriptum, dans Antichità Altoadriatiche, 83 (2016), série Instrumenta inscripta, 6, pp. 39-56.

5. Francis Haskell, History and its Images : Art and the Interpretation of the Past, New Haven, Yale University Press, 1993, pp. 14-20.

6. Marino Marini, Degli aneddoti di Gaetano Marini, Roma, Rome, 1822 ; Pierre Battifol, Gaetano Marini et les collections du Saint-Siège en 1798-1799, dans Bull. Soc. Ant. Fr., 1889, pp. 106-113 ; Henri Leclercq, sub voce "Marini (Gaetano)», dans D.A.C.L., t. X, 2, Paris, 1932, col. 2151-2154 ; Philippe Boutry, Souverain et pontife : recherches prosopographiques sur la curie romaine à l'âge de la restauration (1814-1846), Rome, Collection de l'École française de Rome, 2002, pp. 583-585.

7. Marco Buonocore, «Gaetano Marini e i suoi corrispondenti », Gaetano Marini (1742-1815) protagonista della cultura europea. Scritti per il bicentennario della morte, t. I, Studi e Testi 492, Cité du Vatican, 2015, pp. 105-225, notamment p. 112 et ssq.

8. A. Coppi, op. cit. note 1, p. 90.

9. Ibidem, pp. 107-116.

10. Angelo Mai, Scriptorum veterum nova collectio e Vaticanis codicibus edita ab Angelo Maio, t. 5, v. 5, Cité du Vatican, 1831, pp. XVII-XVIII.

11. Pour la recension des manuscrits de Marini, on peut se reporter à la préface de Giovanni Battista De Rossi à l'œuvre posthume de Gaetano. Marini, Iscrizioni antiche doliari, Rome 1884, pp. III-IX, ainsi qu'à la contribution de Marco Buonocore, Gaetano Marini e la genesi del primo corpus delle iscrizioni cristiane latine e greche, dans Acta XII Congressus Internationalis Epigraphiae Graecae et latinae - Barcelona, 3-8 Septembris 2002, sous la dir. de Marc Mayer i Olivé, Giulia Baratta, Alejandra Guzmán Almagro, Barcelone, 2007, pp. 203-209. Il faut également y ajouter celle des mss. Vat. lat. 9042 à 9060 qui constituent les index analytiques de la recension épigraphique : Danilo Mazzoleni, I codici Vat. lat. 9071-9074, dans op. cit. note 7, pp. 1254-1261.

12. ICUR = Inscriptiones Christianæ Urbis Romæ septimo sæculo antiquiores, (éd. Giovanni Battista De Rossi) vol. I, Rome, 1857-1861, p.XXXIII*. Sur les présupposés et les limites apodictiques de la tradition épigraphique initiée par De Rossi, voir Carlo Carletti, Epigrafia dei cristiani in Occidente dal III al VII secolo : ideologia e prassi, Bari, Edipuglia, 2008, pp. 13-18.

13. H. Leclercq, op. cit. note 6.

14. Marco Buonocore, «Corrispondenze epigrafiche nei codici Vaticani Latini 9042-9060 di Gaetano Marini », Epigraphica, 53, 1991, pp. 216-219 ; M. Buonocore, «Per un'edizione dei codici Vaticani latini 9071-9074 di Gaetano Marini : l'epigrafia cristiana dalle origini fino all'anno mille», Miscellaneae Bibliothecae Apostolicae Vaticanae 8, 2001, pp.45-73; M. Buonocore, "Gaetano marini e la genesi del primo corpus delle iscrizioni cristiane latine e greche ", Acta XII Congressus Internationalis Epigraphiae Graecae et latinae - Barcelona, 3-8 Septembris 2002, Barcelone, 2007, p. 2013-2010.

15. Antonella Ballardini, «Mosaici e pitture medievali di Roma nei codici epigrafici di Gaetano Marini », dans op. cit. note 7, pp. 1594-1655.

16. M. Marini, op. cit. note 6, p. 203.

17. Antonio Manfredi, Andreina Rita, « Notizie sulla Vaticana in età napoleonica. Lettere inedite di Gaetano Marini e Angelo Battaglini (Parigi 1810-1815)» dans op. cit, note 7, pp. 515-585.

18. Ibidem. Lettres de la bibliothèque apostolique vaticane, Arch. Bibl. 52, pt. A., f. IVrv; f. XIIrv, respectivement datées des mois de mars 1812 et mars 1814.

19. Krysztof Pomian, «Una collezione al crepuscolo dei Lumi», dans La collezione Borgia, curiosità e tesori da ogni partent del mondo, Naples, Electa, 2001, pp. 21-29.

20. Ingo Herklotz, «Christliche und klassische Archäologie im sechzehnten Jahrhundert: Skizzen zur Genese einer Wissenschaft ", Die Gegenwart der Altertums. Formen und Funktionen des Altertumbezugs in den Hochkulturen (sous la dir. de Dieter Kuhn Helga Stahl), Heidelberg, 2001, pp. 275-290; Ingo Herklotz, «Archeologia cristiana e archeologia classica nel XVI secolo: riflessioni sulla genesi di una nuova disciplina », La Roma degli antiquari : cultura e erudizione 
tra cinquecento e settecento, Rome, De Luca Editori d'Arte, 2012, pp. 57-66 ; Martine Gosselin, "The Congregation of the Oratorians and the Origins of Christian Archaeology: a Reappraisal ", dans Revue d'histoire ecclésiastique 104, 2, 2009, pp. 471-493 ; Simon Ditchfield, « Text before the Trowel : Antonio Bosio's Roma Sotterranea revisited ", Sacred History. Uses of the Christian Past in the Renaissance World, Oxford, Oxford University Press, 2012, pp. 343-360. Plus nuancée, la position de Massimo Ghilardi, Gli arsenali della fede. Tre saggi su apologia e propaganda delle catacombe romane (da Gregorio XIII a Pio XI), Rome, Aracne; 2006, pp. 13-27.

21. Daniela Mondini, «Séroux d'Agincourt et l'art des premiers chrétiens », Penser l'art dans la seconde moitié du XVIII ${ }^{\mathrm{e}}$ siècle : théorie, critique, philosophie, histoire, (éd.) Villa Médicis, Rome, 2013, pp.549-566; Anne-Orange Poilpré, «Que disent les images chrétiennes de l'identité biblique entre le III et le IV siècle ?», Hortus artium mediev. 20/2, 2014, pp. 666-673.

22. Bien que la question n'ait pas l'objet d'une recension systématique, il convient de signaler les préemptions napoléoniennes à Vérone, notamment celle du célèbre « Testament d'Epictete » (IG XII, 3, 330), comme le signale Margherita Bolla, "Bonaparte e l'archeologia a Verona ", dans cat. d'exp., 1797 : Bonaparte a Verona, sous la dir. de Gian Paolo Marchi, Vérone, Museo di Castelvecchio, 19 septembre 1997-11 janvier 1998, Venise, Marsilio Editori, 1997, pp. 135-145. Pour les réquisitions vaticanes, on peut encore s'en tenir au Regestum Clementis papæ V, Rome, 1886 ainsi qu'aux deux articles de Léopold Delisle, Journal des Savants, 1892, pp. 429-441, pp. 489-501.

23. Arnaldo Momigliano, "Ancient History and the Antiquarian ", dans JWCI, 13, 1950, pp. 283-315, en particulier p. 304.

24. Francesco Maria Torrigio, Le sacre grotte vaticane, Viterbe, 1618, pp. 42-43; Vincenzo Forcella, Iscrizioni delle chiese e d'altri edifici di Roma dal secolo XI fino ai giorni nostri, t. VI, Roma, 1875, n. 429.

25. ICUR I, $141=$ CIL VI, 32002. À partir de maintenant les références épigraphiques des occurrences suppléent du fait de leur exhaustivité à d'autres renvois bibliographiques. Les abréviations en usage renvoient à : CIL : Corpus Inscriptionum Latinarum, Berlin ; CIG : Corpus Inscriptionum Graecarum, Berlin; CLE : Anthologia latina, sive Poesis latinae supplementum (Carmina Latina Epigraphica), Leipsig, 1894-1926 ; ICUR : Inscriptiones Christianæ Urbis Romæ septimo sæculo antiquiores, nouvelle série, Cité du Vatican, 1922-1992; IG: Inscriptiones Graecae, Berlin ; ILCV : Inscriptiones Latinae Christianae Veteres, éd. E. Diehl, Berlin, 1925-1931 ; SEG : Supplementum Epigraphicum Græcum, Leyde, 1923.

26. Ian Gruter, Inscriptiones antiquae totius orbis Romani in corpus absolutissimum redactae ingenio ac cura Jani Gruteri, auspiciis Josephi Scaligeri ac Marci Velseri, Heidelberg, Ex Officina Commelianiana, 1603, p. MCLVII (1162).

27. Charles Pietri, «La mort en Occident dans l'épigraphie latine: de l'épigraphie païenne à l'épitaphe chrétienne, $\mathrm{III}^{\mathrm{e}}-\mathrm{VI}^{\mathrm{e}}$ siècles ", La Maison-Dieu : cahiers de pastorale liturgique, 144, 1980, pp. 25-48.

28. Sabrina Pietrobono, «Fabretti, Raffaele», Personenlexikon zur Christlichen Archäologie : Forscher und Persönlichkeiten vom 16. Bis 21. Jahrhundert, sous la dir. de Stefan Heid et Martin Dennert, t. 1, Ratisbonne, 2012, pp. 467-469.

29. R. Fabretti, Inscriptionum antiquarum quae in aedibus paternis asservantur explicatio et additamentum una cum aliquot emendationibus Gruterianis et indice rerum et verborum memorabilium, Roma, ex officina Dominici Antonii Herculis, 1699.

30. Mario Luni et Gianfranco Gori, 1756-1986. Il Museo Archeologico di Urbino. I - Storia e presentazione delle collezioni Fabretti e Stoppani, Urbin, Quattroventi, 1986, pp. 13-50.

31. Krzysztof Pomian, Collectionneurs, amateurs et curieux. Paris, Venise : $\mathrm{XVI}^{\mathrm{e}}$-XVIII ${ }^{\mathrm{e}}$ siècle, Paris, Gallimard, 1987, pp. 272-275.

32. Alfredo Buonopane, "Scipione Maffei e il suo contributo agli studi di epigrafia romana in Europa », Caesarodunum, 27, 1993, pp. 180-193. 
33. Celle-ci partaient, entre autres, du célèbre testament d'Épictète (CIG II, 2448); Scipione Maffei, Verona illustrata, Verona, 1731, col. 207-211.

34. Giovanni Battista Passeri, Relazione del museo di antiche iscrizioni raccolto nel palazzo apostolico di Urbino dall'eminentissimo sig. card. Stoppani legato l'anno MDCCLVI, Pesaro, 1756, p. III (3). Voir également Ida Calabi Limentani, Scienza Epigrafica. Contributi alla storia degli studi di epigrafia latina, Faenza, Stabilimento Grafico Lega, 2010, pp. 115-133.

35. Giovanni Morello, «Il museo "cristiano" di Benedetto XIV », Bollettino dei Monumenti Musei e Gallerie Pontificie, II, 1980, pp. 54-89; Claudia Lega, «La nascita dei Musei Vaticani : Le antichità cristiane e il museo di Benedetto XIV », dans ibid., XXVIII, 2010, pp. 95-184.

36. Francis Haskell, «Introduzione », Benedetto XIV e le arti del disegno. Atti del convegno internazionale di studi (Bologna 28-30 novembre 1994), sous la dir. de Donatella Biaggi Maino, Rome, Quasar, 1998, pp. 5-11.

37. IG XIV, 156*.

38. ICUR VII, 19933-19935 = CIG IV, 8613.

39. Gaetano Marini, Discorso sopra tre candelabri acquistati da S.P. Clemente XIV, Pise, 1771.

40. Silvia Roettgen, Anton Raphael Mengs 1728-1779 - Das Malerische und Zeichnerische, t. I, Munich, 1999, pp. 318-325 ; S. Roettgen, Anton Raphael Mengs 1728-1779 - Leben und Wirhen, t. II, Munich, pp. 406-416 ; Vittorio Casale, L'affresco di Mengs nella volta della Stanza dei Papiri : i risvolti di una sofferta esecuzione, dans Bollettino dei Monumenti Musei e Gallerie Pontificie XXVI (2007-2008), pp. 157-174.

41. Giuseppe Cozza Luzi, "L'Aula dei Papiri nella Biblioteca Vaticana », Monumenta Papyracae Latina, sous la dir. d'Orazio Marucchi, Rome, 1895, pp. 35-57.

42. Gaetano Marini, I Papiri diplomatici, raccolti ed illustrati dall' abbate Gaetano Marini, Rome, 1805 , p. XXII. Marini visait encore une fois explicitement Winckelmann.

43. Pascal Griener, La République de l'œil, Paris, Odile Jacob, 2010, pp. 162-167.

44. S. Roettgen, op. cit. note 40.

45. Dominique Farout, De la Renaissance à la Restauration : quelques étapes du déchiffrement des hiéroglyphes, dans https://journals.openedition.org/cel/433 [03/12/2018].

46. S. Roettgen, op. cit. note 40.

47. CIL VI, 8506.

48. L'étude des échanges entre l'épigraphiste et le philosophe n'a pas encore fait l'objet d'une étude approfondie. Quelques anticipations sont données par Elena Vaiani, «Raffaele Fabretti, il "Signor Censore »: una polemica antiquaria sui medaglioni di Gaspare Carpegna », Studi Seicenteschi, 46, 2005, pp. 211-228.

49. Maurizio Ferraris, "Monogramme et hologramme à l'époque de Vico ", actes du colloque Giambatista Vico aujourd'hui, Montpellier, Université Paul-Valéry, mai 1994, sous la dir. de Riccardo Pineri, Montpellier, 1996, pp. 123-153.

50. Giovanni Ciampini, Vetera monimenta, Ex typographia Joannis Jacobi Komarek Bohemi, Rome, 1690, t. I, pp.90-105; Walter Otto Fink, «Bemerkungen zu Monogrammen auf einer spätantiken Inkrustationsplatte», Lebendige Altertumswissenschaft. Festgabe zur Vollendung des 70. Lebensjahres von Hermann Vetters, dargebracht von Freunden, Schülern und Kollegen, Vienne, 1985, pp. 290-294.

51. BAV, ms. Vat. lat. 9097, p. 362. Marini précise d'ailleurs que Plutarque se réfère peut-être à la tachygraphie et aux célèbres Notes tironiennes, attribuées à un esclave affranchi de Cicéron dans la vie de Caton le jeune. Comme le distingue Victor Garthausen, Éléments de paléographie grecque (trad. de Charles Cucuel), Paris, 1891, pp. 132-133: les Notes tironiennes simplifiaient les lettres et non les mots.

52. BAV, ms. Vat. lat. 9097, p. 366 ; cit. [Raban Maur], Opera quae reperiri potuerunt omnia in sex tomos distincta, t. VI, Cologne 1626, p. 334. 
53. Ibidem. Pour l'apport de Walafrid, voir Magali Coumert, Raban Maur et son temps, Turnhout, Brepols, 2010, pp. 137-153. La terminologie latine de Walafrid doit, au demeurant, être observée chez Vico dans son De antequissima Italorum sapientiae, dir. Manuela Sanna, Rome, 1710, 2005, Lib I, cap. I, p. 17.

54. Nicolas Oikonomidēs (dir.), Abbreviations in Greek Inscriptions, Papyri, Manuscripts and Early Printed Books, Chicago, Ares, 1974, pp. 15-16.

55. D. Mazzoleni, op. cit. note 11 et M. Buonocore, op. cit. note 11.

56. Supra, note 38 .

57. Gabriel Sanders, "La pérennité du message épigraphique: de la communauté chrétienne élitaire du bas-empire au corps professoral de l'université médiévale de Bologne ", colloque AIEGM La terza età dell'epigrafia - Borghesi 86, Bologne, octobre 1986, Faenza, 1988, pp. 349-414, en particulier p. 352.

58. Ils sont: ms. Vat. Lat. 9072, p. 277, n. 10 (ICUR VIII, 23304), p. 472, n. 13 (ICUR X, 26654a), p. 521, n. 3 (ICUR I, 719) ; ms. Vat. Lat. 9073, p. 591, n. 6 (ICUR X, 27176), p. 635, n. 6 (ICUR III, 8748), p. 652, n. 7 (ICUR VII, 20635), p. 718, n. 5 (ICUR I, 3439) ; ms. Vat. Lat. 9074, p. 899, n. 6 (ICUR VIII, 21610 - CLE 674), p. 913, n. 9 (ICUR ?), p. 913, n. 10 (ICUR I, 51).

59. Ildar Garipzanov, Graphic Signs of Authority in Late Antiquity, Oxford, Oxford University Press, 2018, pp. 1-23.

60. CIL XI ${ }^{1}, 265$.

61. CIL XI ${ }^{1}$, 279. Cf. BAV, ms. Vat. lat. 9107, f. 102r, fiche 6709.

62. CIL XI ${ }^{1}, 283$.

63. $\mathrm{CIL} \mathrm{XI}^{1}, 290 \mathrm{a} / \mathrm{b}$.

64. CIL XI ${ }^{1}, 290 \mathrm{c}$.

65. G. Marini, op. cit. note 42, n. CXIV (114), pp. 172-174 et pp. 328-336.

66. Friedrich Wilhelm Deichmann, «Giuliano Argentario», dans Felix Ravenna, LVI, 1951, pp. 5-26 ; F. W. Deichmann, «I titoli dei vescovi ravennati da Ecclesio a Massimiano nelle epigrafi dedicatorie di San Vitale e di Sant'Apollinare in Classe tramandate da Agnello ", dans Studi Romagnoli, III, 1952, pp. 63-67.

67. Denis Feissel, « Le Préfet de Constantinople, les poids étalons et l'estampillage de l'argenterie au VI et au VII ${ }^{\mathrm{e}}$ s. », Revue numismatique, 28, 1986, pp. 119-142.

68. Corpus iuris civilis, III: Novellae, CXXXI, II [éd. Rudof Schoell, Wilhelm Kroll, Dublin-Zürich, 1972].

69. D. Feissel, «Les édifices de Justinien au témoignage de Procope et de l'épigraphie », dans Antiquité Tardive, 8, 2000, pp. 81-104.

70. Jean-Pierre Caillet, L'évergétisme monumental chrétien en Italie et à ses marges. D'après l'épigraphie des pavements de mosaïque (IV ${ }^{\mathrm{e}} \mathrm{VII}^{\mathrm{e}}$ siècles), École française de Rome, Rome, 1993, pp. 424-425. Il en va de même pour les monogrammes de Grado et Aquilée, Poreć et Pula: voir D. Mazzoleni, Le iscrizioni musive della basilica di S. Eufemia a Grado nel Vat. Lat. 9071 di Gaetano Marini, dans Marmoribus vestita. Miscellanea in onore di Federico Guidobaldi, vol. II, Cité du Vatican, 2011, pp. 923-944 et J.-P. Caillet, op. cit. note 70, pp. 218-257.

71. Ibid., note 69 .

72. Thomas Creissen, «Les clôtures de chœur des églises d'Italie à l'époque romane : état de la question et perspectives », Hortus Artium Medievalium, 5, 1999, pp. 169-181.

73. Nov. Ius., CXXXI, III, note 68.

74. Cat. d'exp., Byzance. L'art byzantin dans les collections publiques françaises, Musée du

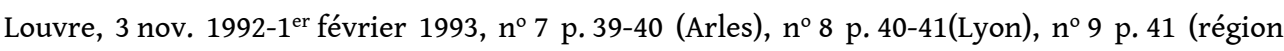
pyrénéenne).

75. A. Ballardini, op. cit. note 15. Marini compare les plus anciens dessins de cette abside avant sa restauration des années 1669-1671 (ms. Vat. lat. 14738, f. 4 - assez proche du ms Windsor RL 9196 de Cassiano dal Pozzo) pour ne retenir que celui d’A. Chacón (ms. Vat.lat. 5407, f. 81). 
76. A. Ballardini, op. cit. note 15. Pour les emprunts théodoriciens de Charlemagne, voir JeanPierre Caillet, L'art carolingien, Paris, Flammarion, 2005, pp. 13-33.

77. Pierre Battifol, «Inscriptions byzantines de Saint-Georges au Vélabre », dans Mélanges d'archéologies et d'histoire, 7, 1887, pp. 419-431; Antonio Muñoz, Il restauro della basilica di S. Giorgio al Velabro in Roma, Rome, 1926, p. 13.

78. Jean Mabillon, Iter Italicum litterarium, Paris, 1687, p. 214. Marini ne retient pas celle, beaucoup plus fautive, d'Anton Francesco Frisi, Memorie storiche di Monza e sua corte, t. III, Milan, 1794, pp. 184-185, pl. XVIII.

79. Xavier Barbier de Montault, «Le trésor de la basilique royale de Monza », dans Bulletin Monumental, 1882, pp. 596-601.

80. Victor Gardthausen, Die griechische palaeographie, Leipsig, 1879, p. 179.

81. I. Garipzanov, op. cit. note 59, pp. 281-282. Pour les principes de psephie et d'isosephie en contexte chrétien, se reporter principalement à Pasquale Testini, Archeologia Cristiana, Bari, 1980, pp. 357-359.

82. BAV, ms. Vat. lat. 9071 , p. 183 , n. 10a/b/c. et ms. Vat. lat. 9074 , p. 1023, n. 6, 7, 8, 11. Philip Grierson - Mark Blackburn, Medieval European Coinage, The Early Middle Ages (5th - 10th centuries), vol.1, Cambridge 1986, pp.190-235, en particulier p. 199 ; Rosamond McKitterick, Charlemagne. The Formation of a European Identity, Cambridge, 2008, pp. 274-275.

83. Michel Rostovtsev - Maurice Prou, Supplément au Catalogue des plombs antiques de la Bibliothèque Nationale, dans Revue Numismatique, 1900, pp. 313-354, n. 997-996.

84. Y. Garipzanov, op. cit. note 59, pp. 256-257.

85. Richard Delbrück, Die Consulardiptychen und verwandte Denkmaeler (Studien zur spaetantiken Kunsgeschichte, 2), Berlin-Leipzig, 1929.

86. Ibid., n. $32=$ CIL V$^{2}, 8120,6$.

87. Ibid., n. 41 = CIL XIII' ${ }^{3}$ 2, 10032.

88. Bartolomeo Borghesi, Oeuvres, vol. VII, Lettres, Paris, 1872, p. 51.

89. R. Delbrück, op. cit. note 85 , n. 59.

90. Anton Francesco Gori, Thesaurus veterum diptychorum consularium et ecclesiasticorum [...], t. II, Florence, 1759, pl. XIX (19).

91. R. Delbrück, op. cit. note 85, n. $19=\mathrm{CIL} \mathrm{V}^{2}, 8120,2$.

92. Ibid., n. 11 = CIL XIII ${ }^{3}, 2,10032$, 3c.

93. Bente Kiilerich, «Symmachus, Boethius and the Consecratio Ivory Diptych», Antiquité Tardive, 20, 2012, pp. 205-215.

94. Lellia Cracco Ruggini, «I dittici tradoantichi nel Medioevo », Il calamo della memoria, IV, 2011, pp. 77-99.

95. BAV, ms. Vat. lat. 9057, ff. 320-323. Voir M. Buonocore, op. cit. note 7, p. 116 ; Ludovico Rebaudo, sub voce «Siauve Étienne-Marie », dans http://www.dizionariobiograficodeifriulani.it [03/12/2018].

\section{RÉSUMÉS}

L'œuvre de Gaetano Marini (1742-1815) est encore largement ignorée de l'historiographie des dernières décennies $\mathrm{du}$ XVIII ${ }^{\mathrm{e}}$ siècle. Il s'agit pourtant d'un épigraphiste, paléographe, archiviste et conservateur de tout premier plan: non seulement pour la qualité de ses recherches, mais 
aussi pour la place qui est la sienne, au Vatican, au cœur des réalisations muséales et des vicissitudes des confiscations napoléoniennes. Dans cet article, le cas des monogrammes chrétiens permet de circonscrire un nouvel aspect de sa contribution à la connaissance de l'imagerie tardo-antique. En effet, bien qu'inédits, les quatre volumes de ses Inscriptiones christianae latince et graeca aevi milliarii de Marini bornent au prisme de l'épigraphie les nuances d'une méthode typologique dont il convient d'apprécier l'ampleur des aspects novateurs vis-à-vis d'un champ des études alors émergent.

The work of Gaetano Marini (1742-1815) is still largely unknown in the historiography of the last decades of the eighteenth century. He was, however, a first-rate epigraphist, palaeographer, archivist and curator: not only for the quality of his research, but also for his place at the Vatican, at the heart of the museum developments and the vicissitudes of the Napoleonian confiscations. In this article, the case of Christian monograms allows us to add a new aspect to his contribution to the knowledge to the imagery of Late Antiquity. Although they were unpublished, the four volumes of his Inscriptiones christianae latince et graeca aevi milliarii see through the prism of epigraphy the nuances of a typological method whose innovations should be appreciated vis à vis a then emerging field of studies.

\section{INDEX}

Mots-clés : Fabretti, Maffei, Marini, musées du Vatican, lapidarium, monogrammes, épigraphie, inscriptions latines, antiquité tardive, archéologie chrétienne

Keywords : Fabretti, Maffei, Marini, Musei Vaticani, lapidarium, monograms, epigraphy, Latin inscriptions, Late Antiquity, Christian archaeology

\section{AUTEUR}

\section{ISABELLE MATHIAN}

Ancienne élève de l'École du Louvre et membre de son équipe de recherche, Isabelle Mathian a soutenu en 2016 une thèse de $\mathrm{III}^{\mathrm{e}}$ cycle sous la direction de Jean-Pierre Caillet intitulée Le nu tardo-antique et ses prolongements médiévaux : genèse et devenir des figures de Daniel et d'Adam et Ève. Ses recherches portent sur l'iconographie tardo-antique et proto-médiévale ainsi que sur les ressors de l'historiographie tardo-antique.

***

A former student of the École du Louvre and member of its research team, Isabelle Mathian defended a thesis under the supervision of Jean-Pierre Caillet entitled Le nu tardo-antique et ses prolongements médiévaux : genèse et devenir des figures de Daniel et d'Adam et Ève in 2016. Her research focuses on the iconography of Late Antiquity and the early Middle Ages as well as on the problematics of the historiography of Late Antiquity. 